\title{
Macroscopic Quantum Effects in Disordered Systems
}

\author{
M. Ya. Azbel', Tel Aviv \\ A. Hartstein, Yorktown Heights \\ and D.P.Di Vincenzo, Cornell
}

A characteristic trend in solid state physics is the roughly exponential decrease with time in available temperature and the size of devices - see Figs. 1 and 2: during the last two decades, temperatures have dropped by four orders of magnitude and the size of commercial devices by two orders of magnitude! Of course in Fig. 1, record temperatures are presented, but $50-100 \mathrm{mK}$ is not out of the way in the laboratory. The development of micron and submicron devices, in part related to advances in computer technology, has resulted in insulating thicknesses becoming as small as $100 \AA$. This trend to subkelvin temperatures and to micron and submicron devices leads to qualitatively new physical properties.

At temperatures where thermal fluctuations may be neglected, the Schrödinger equation is applicable to the whole macroscopic system. It predicts the eigenstates, which depend on the microscopic structure of the system and are therefore individual for each given system. In this respect, a macroscopic system resembles a giant "superatom" with a specific energy spectrum. Its spectroscopy should also be as specific as the familiar spectroscopy of atoms

Thus, at a sufficiently low temperature $T$, any system becomes a macroscopic quantum system. The higher the temperature, the smaller the system must be. In micron and submicron MOSFETs, the Si inversion layers may fall into the range in question. The behaviour of such systems is very unusual, as demonstrated in e.g. experiments by Fowler, Hartstein and Webb (see Fig. 3). The plot can be viewed as representing the conductance $G$ of a sample $(10 \mu \mathrm{m}$ long, $100-300 \AA$ wide and $50-80 \AA$ thick) against the number $N$ of electrons in the sample. The electron density $n$ in the experiment is controlled by the applied gate voltage $V$ and $N$ varies from $\approx$ and molecules.

$10^{4}$ to $1.5 \times 10^{4}$. The corresponding Fermi energy changes from $\approx 20 \mathrm{meV}$ to $\approx 30 \mathrm{meV}$. Clearly $G(N)$ exhibits unconventional features:

(i) The envelope dependence of the conductance on the electron density is exponential rather than linear.

(ii) The addition of only a few extra electrons may change the conductance by two orders of magnitude.

(iii) The peaks in $G$ are extraordinarily sharp and narrow.

(iv) The overall structure of $G$ appears chaotic. Indeed, the peaks seem randomly situated, whereas they are unique to any given sample, and are perfectly reproducible even after moderate temperature cycling.

(v) The temperature dependence of maximum (peak) and minimum (valley) conductances $G$ follow the variable range hopping law predicted by Mott:

$$
\text { In } G \sim-\left(T_{0} \cdot / T\right)^{1 / 2}
$$

as shown in Fig. 4.

The reference temperature $T_{0}{ }^{*}$ relating to peaks is itself a chaotic function of the electron density superimposed on an overall exponential envelope that is dependent on $n$. For instance, the highest peaks, observed by A. Hartstein et al. have values of $T_{0}{ }^{*}$ shown in Table 1.

Such "reproducible chaos" is a characteristic feature of a number of small systems at low-temperature. To understand these results, first consider quantum scattering by two one-dimensional barriers (we choose rectangular for simplicity) with a potential well in between (Fig. 5). Suppose the transmission coefficients of the barriers are $t_{1}$ and $t_{2}<t_{1}$ $\ll 1$. At most energies, the total transmission coefficient $t_{12}$ is given by:

$$
t_{12} \sim t_{1} t_{2}
$$

The transmission is little dependent on the well, and the barriers are effectively combined, a result which is physically natural. In a wide and high barrier, the particle probability density $\rho$ decreases as in an infinitely wide barrier, i.e. expo-

Table 1 - A typical set of results showing the chaotic behaviour of $T_{0}{ }^{\circ}$

\begin{tabular}{|c|c|c|c|c|c|c|c|c|c|c|c|c|}
\hline $10^{-12} n$ & 1.6247 & 2.093 & 2.4188 & 2.9000 & 3.2130 & 3.2762 & 3.3072 & 3.3907 & 3.4733 & 3.5239 & 3.6244 & 3.7924 \\
\hline$T_{0}^{*}(\mathrm{~K})$ & 8.8 & 4.9 & 8.6 & 1.8 & 6.6 & 4.0 & 1.7 & 4.3 & 2.3 & 1.9 & 2.7 & 1.6 \\
\hline
\end{tabular}

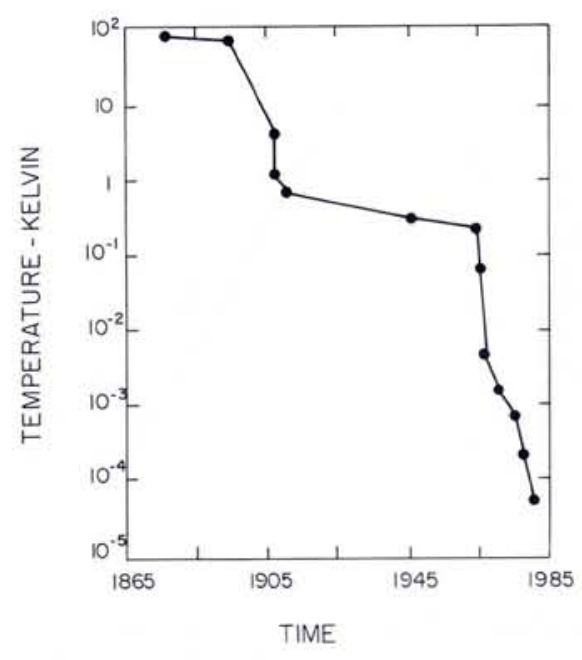

Fig. 1 - Experimentally available temperature vs. year, by R.A. Webb.

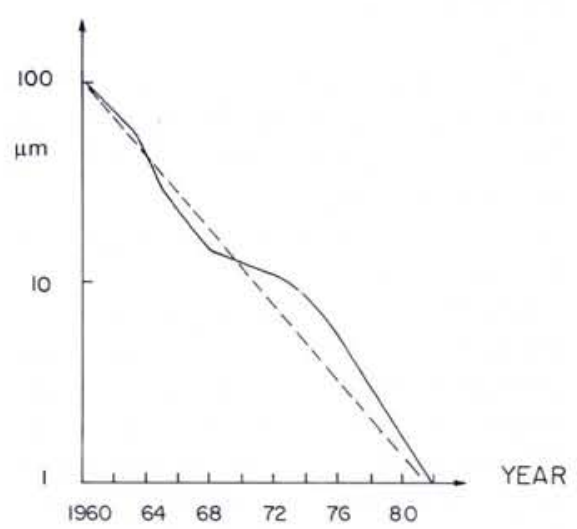

Fig. 2 -Commercial device size vs. year, by R.A. Webb.

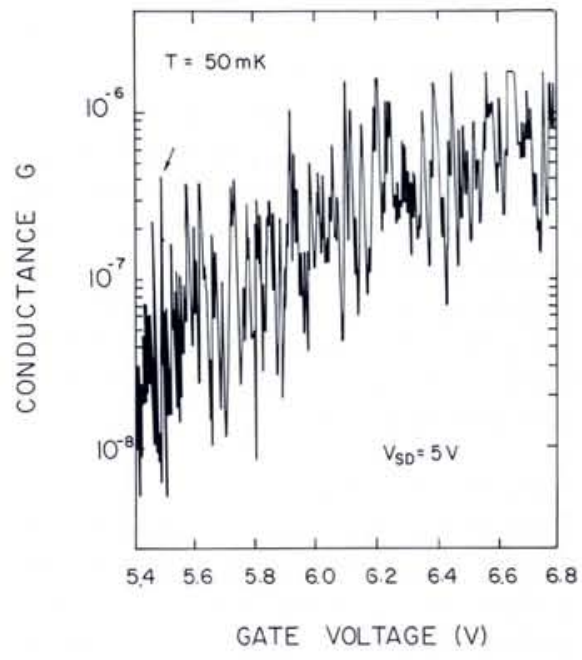

Fig. 3 - Conductance $G$ of a Si inversion layer vs. gate voltage $V$, according to $A$. Hartstein, A.B. Fowler and R.A. Webb lunpublished). Source - drain voltage drop is 5 $\mu \mathrm{V}$; temperature is $T=50 \mathrm{mK}$. (Gate voltage determines the electron density $n_{e}=$ $\left.7.3 \times 10^{11} \times(V-1.1) \mathrm{cm}^{2}.\right)$ 


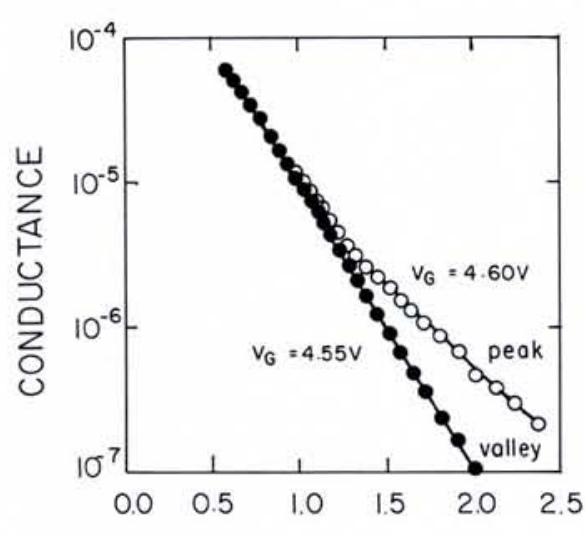

$T^{-1 / 2}$

Fig. 4 - Temperature dependence of conductance for a peak (gate voltage $4.60 \mathrm{~V}$ ) and a valley (gate voltage $4.55 \mathrm{~V}$ ) by A.B. Fowler, A. Hartstein and R.A. Webb; Physica, 117B-118-B (1983) 661. The sample is the same as in Fig. 3.

nentially: by a factor $1 / t_{1}$ in the first and $1 / t_{2}$ in the second (Fig. 5 , dashed line). The ratio of the probability densities at the extreme barrier edges, which is the transmission coefficient is then given by Eq. (2).

However, there is a narrow range of energies, for which Eq. (2) is not valid, notably when the potential well between infinitely wide barriers yields eigenstates. In this case the corresponding $\rho$ decreases exponentially in both barriers outwards. If the barriers are finite, tunnelling of a particle can take place, the probability of which is determined by the "easier" barrier. Assuming $t_{1} \gg t_{2}$ the lifetime $\tau$ inside the well is $1 / t_{1}$, so according to the uncertainty principle, the half-width $\delta \varepsilon$ of an eigenstate is

$$
\delta \varepsilon \sim h / \tau \sim t_{1}
$$

(likewise if $t_{2} \gg t_{1}$, then $\delta \varepsilon \sim t_{2}$ ).

A simple calculation demonstrates that in this energy region, $\rho$ behaves in a way similar to that for infinitely wide barriers, i.e. it decreases towards the outer edges of the barriers, by $t_{1}{ }^{-1}$ in the first and by $t_{2}^{-1}$ in the second (Fig. 5, solid line). So the total transmission coefficient $t_{12}$, which is the ratio of the $\rho$ s at the outer-edges, is:

$$
t_{12} \sim t_{2} / t_{1} \text {, if } t_{2}<t_{1}
$$

(and $t_{1} / t_{2}$, if $t_{1}<t_{2}$ ).

Thus, in the immediate vicinity of a well eigenvalue $\varepsilon$, the transmission resonantly increases by a factor $t_{2}{ }^{2}$. Note that identical barriers yield $t_{12}=1$; a periodic array of barriers leads to the Bloch theorem and energy bands.

Now consider the general case of a random one-dimensional (1D) array of barriers. If an incident particle has an energy different from any eigenvalue of the system, the total transmission coef- ficient $t$ is given by $t \sim t_{1} t_{2} \ldots t_{N}$, where $t_{1}, t_{2}, \ldots t_{N}$ are the transmission coefficients of successive barriers. Thus,

$$
\begin{aligned}
& t \sim \exp \left(\ln t_{1}+\ldots+\ln t_{N}\right) \\
& \approx \exp (N<\ln t>)
\end{aligned}
$$

where $<>$ denotes the mean value. Since the number of barriers $N$ is proportional to the length $L$ of the system, in any $1 \mathrm{D}$ random system, as $L \rightarrow \infty$,

$$
t \sim \exp \left(-L / L_{0}\right)
$$

where $L_{0}$ is the "localization length". Landauer demonstrated already in 1970 that in 1D, the zero-temperature conductance is proportional to the transmission coefficient:

$$
G \sim\left(2 \mathrm{e}^{2} / h\right) t
$$

so we come to the well-known paradoxical result: the residual conductance of a 1D random system is exponential rather than linear with length: resistances in series multiply rather than add.

Now suppose the energy $\varepsilon$ of an incident particle equals one of the eigenvalues. All potential barriers to the left and to the right of the effective potential well of the eigenstate can be considered together (as $\varepsilon$ is an "ordinary" energy with respect to those regions). Their respective transmission coefficients are then given by:

$t_{1} \sim \exp \left(-L_{1} / L_{0}\right), t_{2} \sim \exp \left(-L_{2} / L_{0}\right)$ (7) and the overall resonant transmission coefficient from Eq. (4), by

$$
t \sim \exp \left(-\left|L_{1}-L_{2}\right| / L_{0}\right)
$$

The eigenstate half-width $\delta \varepsilon$ moreover is seen to be

$$
\begin{aligned}
\delta \varepsilon \sim t_{1}+t_{2} & \sim \exp \left(-L_{1} / L_{0}\right) \\
& +\exp \left(-L_{2} / L_{0}\right)
\end{aligned}
$$

In a random system, eigenstates are localized at random places, so $L_{1}$ and $t$ change randomly with energy.

Now, if the incident beam is non-monochromatic, and its energy width $\varepsilon_{\mathrm{nm}}$ $\gg \delta \varepsilon$, then only a fraction $\delta \varepsilon / \varepsilon$ of the particles squeeze into the resonance width. This leads to an effective transmission coefficient $t_{\mathrm{nm}} \approx t\left(\delta \varepsilon / \varepsilon_{\mathrm{nm}}\right)$. Taking again $t_{1}>t_{2}$, then, from Eq. (3) $\delta \varepsilon \sim t_{1}$ so that from Eq. (4) we have $t_{\mathrm{nm}} \sim t_{1}\left(t_{2} / t_{1}\right)$, i.e. $\quad t_{\mathrm{nm}} \sim t_{2}$ and so is independent of $t_{1}$.

So far we have been assuming that the temperature is so low as to be negligible, but if we are to understand the effect of a finite temperature, we must consider localized eigenstates in more detail. Whilst the probability density $\rho$ decreases exponentially in space outside the localization region, in a random system such regions are randomly situated, and their positions and energies are in no way correlated. In general, therefore, eigenstates of similar energy are far from each other in space and there is little overlap of their probability densities. On the other hand, eigenstates with a noticeable overlap are likely to have significantly different eigenenergies.

At low temperatures, a particle hops from one eigenstate to another by finding the best compromise between the high thermal population (of eigenstates close in energy) and high probability density overlap (of eigenstates close in space). Mott demonstrated that the resulting transition probability $w \sim \exp$ $\left[-\left(T_{0} / T\right)^{1 /(D+1)}\right]$, where $D$ is the dimensionality; the corresponding average time of a single inelastic hop from one energy to another $\tau_{\text {in }} \sim w^{-1}$. Since localization regions are randomly situated, successive hops form a random walk. Hence, the corresponding diffusion time to get from one place to another is $\tau_{\mathrm{D}} \sim$ $\tau_{\text {in }} \sim w^{-1 / 2}$. The uncertainty principle then yields the modified eigenstate halfwidth $\delta \varepsilon$ as $\sim w^{-1 / 2}$. So, by Eq. (10), the strongest resonances lead to

$t_{\mathrm{nm}} \sim \delta \varepsilon / k_{\mathrm{B}} T_{\mathrm{O}} \sim \exp \left[-1 / 2\left(T_{\mathrm{O}} / T\right)^{1 /(D+1)}\right]$

(The factor $1 / k_{\mathrm{B}} T_{0}$, with $k_{\mathrm{B}}$ being the Boltzmann constant, follows from a somewhat more accurate derivation.) According to Eqs. (1, 6 and 11$), T_{0}{ }^{\circ} \approx$ $T_{0} / 4$ in 1D. More specifically, $T_{0}{ }^{\circ}$ as $t$ in Eq. (8), depends on the localization length of the corresponding eigenstate. Hence, it is a "chaotic" function of the eigenenergy, and even for the strongest resonances $T_{0}{ }^{\circ} \approx T_{0} / 4$ only on average. This conclusion is in good agreement with experiment ${ }^{1}$ ).
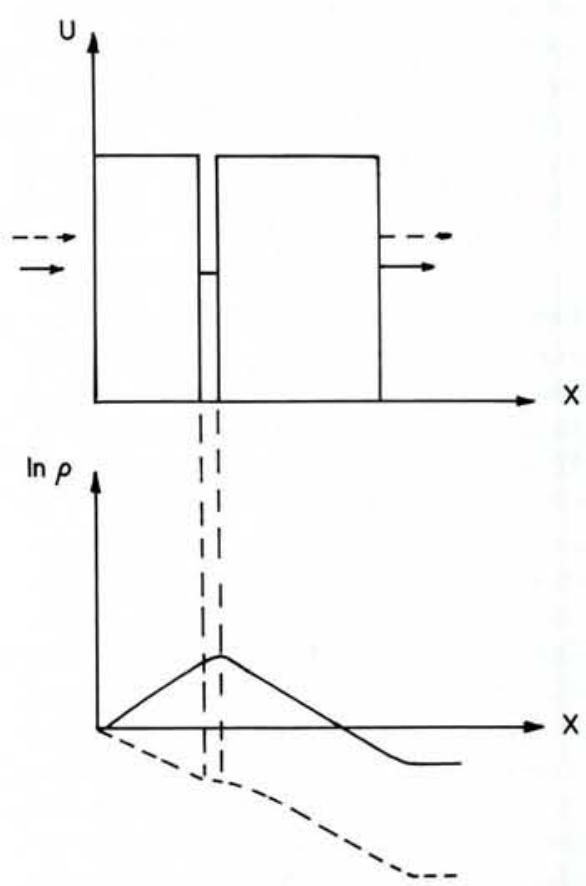

Fig. 5 - Transmission through two onedimensional rectangular potential barriers (above); $U$ is potential energy against coordinate $x$. Solid line corresponds to an eigenenergy, dashed line to an "ordinary" energy. The probability density $\rho(x)$ for both cases is presented by the lower plot. 
The resonances survive at finite temperatures so long as the eigenenergies are clearly distinct from each other. This holds if an eigenstate width is small compared to the mean distance $\Delta \varepsilon$ between the energy levels:

$$
\delta \varepsilon<\Delta \varepsilon \sim 1 / \rho_{D}(\varepsilon) V
$$

where $\rho_{D}(\varepsilon)$ is the $\mathrm{D}$-dimensional density of states, and $V$ is the volume. Thus by Eqs. (11, and 12), resonances can be observed, when

$$
T<T_{0} /\left[2 \ln \left(\rho_{D} V T_{0}\right)\right]^{D+1}
$$

Resonances may survive even when $k_{\mathrm{B}} T \gg \Delta \varepsilon$. This happens when the eigenstates, separated by $\Delta \varepsilon$, are far away from each other compared to $L_{0}$. Then their probability densities have almost no overlap, and transitions between them are unlikely. The observed resonance width in this case is $\sim k_{\mathrm{B}} T$. These conclusions are also consistent with experiment.

Thus, a simple physical picture gives us at least a qualitative understanding of some basic features of the observed resonances. Naturally, eigenstates exist in any closed system without energy dissipation. Under certain conditions, random imperfections may localize electromagnetic, ultrasonic, hydrodynamic, waves, etc., giving rise to strong and narrow resonances in a variety of systems provided they are small enough.

The ongoing decrease in achievable temperature and device size will certain-

10 - 21 APRIL 1985

GEILO, NORWAY

\section{NATO ADVANCED STUDY INSTITUTE ON}

Scaling Phenomena in Disordered Systems

TOPICS OF LECTURES covering both theory and experiments

- Scaling concepts in phase transitions and aggregation processes

- Fractal concepts in colloids, gels, polymers and porous media

- Aggregation, percolation and diffusion

- Dynamics of fractal systems

- Cellular automata

Open to participants from all countries. Deadline for application is February 1st 1985. Information:

Gerd Jarrett, Institute for Energy Technology, POB 40, N-2007 Kjeller, Norway

or Roger Pynn, Institut Laue-Langevin,

156X Centre de Tri, F-38042 Grenoble Cedex, France

ly make such manifestations of the quantum properties of macroscopic systems more and more common and significant. The implications of resonances are two-fold. On the one hand, one must be aware of irreproducible behaviour from sample to sample in small low dissipation systems, on the other, such behaviour may in the future allow us to "individually tailor" devices: for instance, a certain current-voltage characteristic. Then one may calculate the potential, which leads to this characteristic, and generate the potential by artificially placed "impurities", which may themselves have macroscopic dimensions. The investigation of macroscopic quan- tum systems has just started. Naturally, it is difficult to foresee their practical im plications more specifically until more detailed experimental and theoretical studies have been undertaken.

\section{BIBLIOGRAPHY}

Fowler A.B., Hartstein A. and Webb R.A., Phys. Rev. Lett. 48 (1982) 196, and Physica 117B-188B (1983) 661.

Hartstein A., Webb R.A., Fowler A.B. and Wainer J.J., Surf. Sci.

Kwasnick R.F., Kastner M.A., Melngaillis J. and Lee P.A., Phys. Rev. Lett. 52 (1984) 224.

Azbel M.Ya., Phys. Rev. B28 (1984) 4106

Azbel M.Ya., Hartstein A. and Di Vincenzo D.P., Phys. Rev. Lett. 52 (1984) 1641.

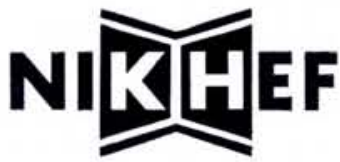 \\ The nuclear physics section of the National Institute for Nuclear Physics and High-Energy Physics (NIKHEF-K) has an opening for a \\ Postdoctoral Research Associate in Theoretical Nuclear Physics}

starting September 1985.

The appointment is for a maximum of two years.

The institute operates a $500 \mathrm{MeV}$ high duty cycle linear electron accelerator to carry out a research program in nuclear structure and nuclear reactions using electron scattering, photonuclear reactions and experiments with low energy pions and muons.

Applicants are expected to have experience in areas of nuclear theory related to the experimental program.
Further information can be obtained from the Scientific Director Prof. Dr. G. van Middelkoop (tel. 020-5922163) or from Dr. J.H. Koch (tel. 020-5922171).

Applications, including a resume and the names of three referees should be sent before Jan. 15, 1985 to:

the Scientific Director, NIKHEF-K, P. O. Box 41882 , NL-1009 DB Amsterdam, The Netherlands. 